\title{
German Curriculum in Interventional Neuroradiology-How Many Interventionalists are Necessary? Did We Need Support from Other Societies for Stroke Treatment?
}

\section{A. Berlis}

Published online: 20 November 2015

C Springer-Verlag Berlin Heidelberg 2015

\section{Introduction}

The German Society of Neuroradiology (DGNR) and the German Society of Interventional Radiology and Minimally Invasive Treatment (DeGIR) in cooperation with the German Society of Radiology (DRG) run a common system for training to acquire additional qualification which exceeds education for general radiology (Modules A-E) and additional education in neuroradiology (Module F). Modules A-D were started in 2009 by the DeGIR and the first steps for a neurointerventional module were made in 2010 and finished with Module E (Recanalization) and F (Embolization) in September 2012.

A board-certified radiologist needs 5 years of education and another 3 years to obtain the subspecialty in Neuroradiology. Specialization in Neuroradiology in Germany requires the following minimum number of interventional neuroradiological procedures: ten recanalizing interventions, ten occluding interventions, and ten percutaneous treatments or biopsies in vascular malformations, tumors, or pain. The number in neurointerventional procedures has continued to rise in the past 15 years. The reason is the now well-established stroke and aneurysm treatment due to the level 1 evidence for both treatment modalities. As a consequence, additional qualification is mandatory to improve quality, including morphological and clinical results, as well as to minimize complications in neurointerventional procedures. Therefore, a dedicated educational program

\footnotetext{
A. Berlis $(\square)$

Klinikum Augsburg,

Stenglinstr. 2,

86156 Augsburg, Germany

e-mail: ansgar.berlis@klinikum-augsburg.de
}

that includes practical aspects in the catheterization laboratory, theoretical knowledge of anatomy and material, and practical skills in neurointerventions is required. All these are based on the specification in radiology and subspecialization in neuroradiology which is important and essential for treatment indication. What is important for endovascular stroke treatment and who is qualified to perform this treatment? Skills in using catheters and microcatheters are certainly necessary, but not sufficient in themselves. Knowledge of the treated organ is also essential.

The DeGIR/DGNR concept is structured training with three levels of certification: Level 1 -basic qualification in interventional radiology (IR), Level 2-specialized training in interventional radiology (IR) and interventional neuroradiology (INR), and Level 3-qualification as a teaching and training institution. The DeGIR/DGNR concept aims to qualify interventional radiologists and interventional neuroradiologists by structuring the training of interventional procedures to guarantee in-depth knowledge and broad skills that are required in clinical practice. The DeGIR/ DGNR concept supports initiatives of the CIRSE and the ESNR aiming for quality improvement, for example, the EBIR certificate and the ESNR certificate as provided by the European Board of Interventional Radiology.

Level 1-basic qualification in interventional radiology. This course provides in-depth understanding of IR techniques. Level 1 students should be superior to normal residents in Radiology in applying special knowledge in Interventional Radiology/Neuroradiology. The condition to obtain Level 1 is a board-certified Radiologist with at least 30 continuing medical education (CME) points within 2 years in courses/ workshops/seminars training material and principles of IR/ INR techniques. The courses should be certified by DeGIR/ DGNR. Basic knowledge about anatomy relevant for IR and INR as well as basic knowledge about clinical, pathologi- 
cal, and pathophysiological conditions of states and diseases being diagnosed and treated with IR or INR are required.

Level 2-Specialized Training in Interventional Radiology/Neuroradiology. The purpose of training is perfect control of vascular recanalization, embolization of aneurysms, embolization of arteriovenous (AV) malformations, and computed tomography (CT)-controlled percutaneous interventions. Various modules are available: Module Atechniques of angioplasty and recanalization; Module Btechniques of vascular occlusion (embolization) with coils, fluids, particles, plugs, etc.; Module C-suited for diagnostic punctures, percutaneous pain treatment, vertebroplasty, drainages, percutaneous transhepatic cholangiography (PTCD), bile ducts, transjugular intrahepatic portosystemic shunt (TIPPS), gastrostomy, port, etc.; Module D-for oncologic procedures including transcatheter arterial chemoembolisation (TACE) or other tumor specific embolization, ablation, percutaneous treatment of tumors. The Neuromodule is divided into Module E for neurovascular recanalization (percutaneous transluminal angioplasty/stent of extracranial/intracranial arteries, supra-aortal arteries, mechanical recanalization in stroke patients, intra-arterial thrombolysis) and Module $\mathrm{F}$ for neurovascular embolization (intracranial and spinal aneurysms, cranial and spinal vascular malformations, tumors).

Qualifying for Level 2 requires practice in Interventional Radiology or Neuroradiology after passing board certification for Radiology. Module $\mathrm{F}$ also requires board certification for Neuroradiology. Application for Modules A-F requires membership in DRG and DeGIR, Modules E and $F$ of level 2 require additional membership in DGNR. The conditions to obtain Modules $\mathrm{E}$ and $\mathrm{F}$ of level 2 require participation in DeGIR/DGNR-certified courses with the achievement of $30 \mathrm{CME}$ points per module. Module E could be reached by radiologists as well as neuroradiologists with certification of at least 100 independently performed treatments in Module $E$ with at least 30 intracranial and at least 30 extracranial procedures. Module F should be reached exclusively by neuroradiologists. The application form must be accompanied by the required 100 independently performed interventions with at least 50 intracranial procedures which are performed under supervision of a certified DeGIR/DGNR teacher in a qualified institution according to Level 3 requirements. Level 2 postulates advanced knowledge in anatomy and in clinical, pathological, and pathophysiological conditions of states and diseases being diagnosed and treated with IR or INR. Furthermore, advanced knowledge and skills of techniques, indications, and results of all imaging modalities are mandatory. Level 2 includes advanced knowledge about material, contrast agents, and drugs and skills in life-supporting measures as well as advanced knowledge and skills in techniques of sedation and analgesia as applied in IR/INR. Regular participation in clinical-radiological conferences (at least once a week) is assumed. The conditions to obtain Level 2 is at least $30 \mathrm{CME}$ credits in courses/workshops/seminars training material and principles of IR/INR techniques and after completing the requirements for Modules $\mathrm{E}$ and $\mathrm{F}$, oral and written tests which start on 1 January 2016.

Requirements to be acknowledged as a DeGIR/DGNR Teaching and Training Institution (Level 3) in Interventional Radiology or Neuroradiology are that training in IR und INR is possible at the home institution as in other qualified institutions. The teaching institution should include in addition to IR/INR other medical disciplines (e.g., angiology, vascular surgery, cardiology, cardiac surgery, neurology, neurosurgery, and anesthesiology) that allow interdisciplinary case discussions and conferences. The institution should provide comprehensive training in Radiology (or Neuroradiology for Module F) and should participate in the DeGIR QS documentation. The application should include a description of structures; statistics of interventions grouped according to the modules, proof of neurosurgery for Module $\mathrm{F}$, proof of 50 interventions for Modules $\mathrm{E}$ and $\mathrm{F}$ within 12 months prior to application. Reevaluation and recertification takes place after a few years.

German neuroradiologists have had experience in endovascular stroke treatment for about 35 years. In the first two decades, fibrinolytics were exclusively used. In the late 1990s, the first mechanical recanalization devices were examined, whereby German neuroradiologists were some of the most active interventionalists worldwide. Parallel to the development of new devices, interest in CT and magnetic resonance (MR) stroke imaging increased and dedicated protocols for patients qualifying for intra-arterial treatment came into existence. Nevertheless, emergency imaging of the brain is recommended before initiating any specific treatment for acute stroke, whereas the benefits of additional imaging beyond CT and CTA or MR and MRA are still not known.

Today, substantial new high-quality evidence regarding the clinical efficacy of endovascular treatments for acute ischemic stroke has become available. In 2015, the following trials were published or presented: MR CLEAN, SWIFT PRIME, EXTEND IA, ESCAPE, REVASCAT, THERAPY, THRACE. The efficacy of intra-arterial therapy in acute ischemic stroke has become established and is now part of national and international stroke guidelines. The patients should receive mechanical thrombectomy with a stent retriever approved by local health authorities if they meet specific criteria (Grade A, Level 1a evidence). Other thrombectomy or aspiration devices approved by local health authorities may be used upon the neurointerventionalist's discretion if rapid, complete, and safe revascularization of the target vessel can be achieved (Grade C, Level 2a evidence). 
With a 35-year history of endovascular stroke treatment, we are all happy to have an evidence-based solution for the treatment of patients with severe ischemic stroke. Up to this year, we had an individual treatment situation. However, we now have a situation asking for a solution to treat all patients who would be eligible for this treatment modality. Fortunately, we started an educational concept in 2012 and we now have about 150 interventionalists with Module E certification and about 110 with Module F certification. We are happy to be present in all 107 German transregional stroke units $(85 \%$ neuroradiological and $15 \%$ radiological interventionalists). It is possible to treat endovascular stroke $24 \mathrm{~h}$ and 7 days in 127 hospitals in Germany. This allows comprehensive endovascular stroke treatment throughout nearly all of Germany and corresponds to the differentiation of regional and transregional stroke units and their allocation of responsibilities of the German Stroke Society (DSG) and German Society of Neurology (DGN). This network must continue to improve in the next few years and the availability of stroke interventionalists in smaller hospitals would be most desirable. However, we have to keep in mind that interventionalists should be trained and skills have to be trained over the time. Every hospital with $24 \mathrm{~h}$ mechanical stroke treatment must have at least 2-3 neurointerventionalists. In Germany, there are about 270,000 stroke patients per year and about 13,000 patients would be candidates for mechanical thrombectomy. If a hospital performs 50 mechanical thrombectomy procedures per year then every neurointerventionalist would perform 16.5 procedures per year or about 1 per month! Thus, 50 cases and 13,000 possible candidates means treatments at 260 sites. A German survey regarding endovascular stroke treatment in Germany demonstrates that $65 \%$ of the centers treat more than 50 patients per year. On the other hand, 50 mechanical thrombectomies translates into 800-1000 stroke patients because only $5-10 \%$ of all stroke patients qualify for mechanical thrombectomy. The main question is how many cases are necessary to maintain high quality.

Is there a necessity for other interventional specialists to support neurointerventionalists in stroke treatment? The answer is clear: No. First of all, there is comprehensive stroke treatment available. Second, structured education for endovascular stroke treatment is available in Germany. And third, stroke treatment is not only recanalization and removal of a clot. The decision to treat is based on an interdisciplinary discussion including imaging, stroke severity, comorbidity, etc.

In conclusion, 35 years ago Germany started with endovascular stroke treatment. Currently, we have an excellent structure combining skills, mechanical thrombectomy, and stroke units. Within the next few years and increasing number of stroke cases, we will be able to educate more neurointerventionalists to improve availability in the endovascular stroke network. It is a large challenge, but the last 3 years have shown that DeGIR and DGNR have already worked out a well-running concept. Our aim is to treat acute ischemic stroke in certificated stroke units with certified interventionalists throughout Germany. 